Highly Energetic Physical Processes and

Mechanisms for Emission from Astrophysical Plasmas

IAU Symposium, Vol. 195, 2000

P. C. H. Martens, S. Tsuruta, and M. A. Weber, eds.

\title{
Gamma-ray Bursts and Afterglow
}

\author{
R. Sari \\ Theoretical Astrophysics 130-33, California institute of Technology, \\ Pasadena CA 91125, U.S.A.
}

\begin{abstract}
The origin of GRBs has been a mystery for almost 30 years. Their sources emit a huge amount of energy on short time scales, and the process involves extreme relativistic motion with a bulk Lorentz factor of at least a few hundred. In the last two years, "afterglow" emission in Xray, optical, IR, and radio was detected. The afterglow can be measured up to months and even years after the few-seconds GRB. We review the theories for the $\gamma$-ray emission and its afterglow, and show that these are strongly supported by observations. A recent detection of optical emission simultaneous with the GRB agrees well with theoretical predictions and further constrains the free parameters of the models. We discuss the evidence that some of the bursts are jets and discuss the prospects of polarization measurements.
\end{abstract}

\section{Extreme Relativistic Motion and the Generic Picture}

The phenomenon of GRBs was discovered almost thirty years ago by the Vela defense satellites (Klebesadel, Strong, \& Olson 1973). Today, the largest catalog of GRBs (Paciesas et al. 1999) is due to the instrument BATSE onboard the Compton Gamma-ray Observatory. BATSE observes about one burst per day, and more than two thousands bursts have been observed by now.

The spectrum of GRBs is well described by a broken power law and usually peaks between 100-400 keV (Band et al. 1993). In strong bursts, high-energy power law tails extending up to $200 \mathrm{MeV}$ were seen, and several photons of a few $\mathrm{GeV}$ were detected in the most extreme case. On the average, the highenergy tail is characterized by $\nu F_{\nu} \sim \nu^{-0.25}$. The durations of the GRBs vary significantly, mainly between a few milliseconds to a few hundred seconds. The duration distribution is bimodal: about a quarter of the bursts are "short", lasting less than 2 seconds, while the majority are "long". One of the striking properties of GRBs is their erratic temporal structure. While only a few bursts are smooth, most of them vary over a time scale $\delta t$ which is much shorter than the burst's duration $t$. In many bursts, the ratio $N \equiv t / \delta t$ is a hundred or more.

The distribution of bursts over the sky, as obtained by BATSE, is extremely uniform. Specifically, there is no excess of bursts in the direction of the galactic plane. The simplest explanation is that the bursts originate from cosmological distances. By now, the cosmological distance scale is well established since redshifts have been measured for more than a handful of bursts. 

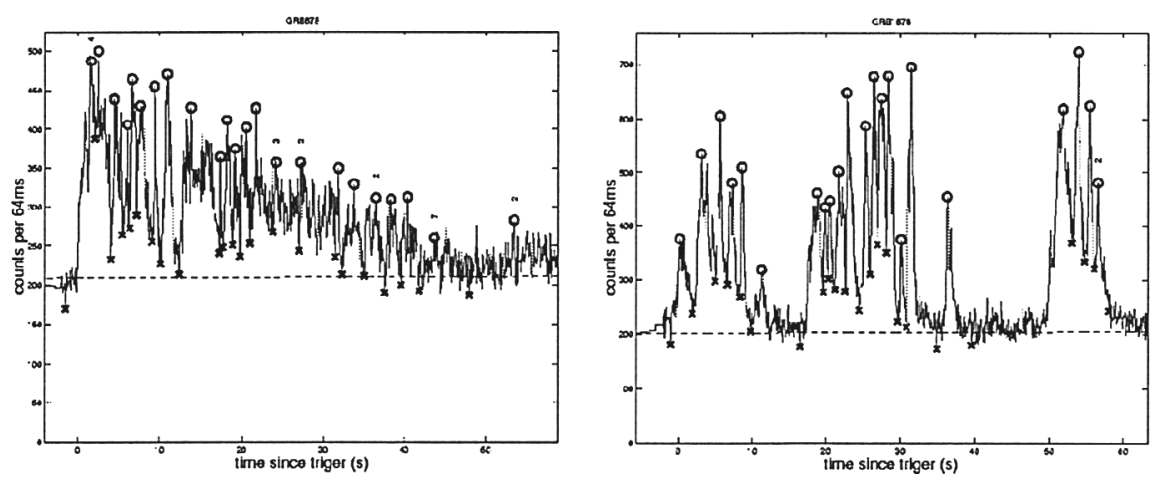

Figure 1. Two variable bursts as measured by BATSE. Statistically significant peaks are circled. The dashed line is the background level.

The large (cosmological) distance to the bursts, combined with the relatively large fluence observed at earth (more than $10^{-6} \mathrm{erg} / \mathrm{cm}^{2}$ ) implies that the energy released in the event is huge, with a record of $3 \times 10^{54} \mathrm{erg}$ (GRB 990123, e.g., see Kulkarni et al. 1999a). This huge energy, together with the short variability time scale, places the GRB phenomenon as the most extreme in the universe.

The extreme characteristics of GRBs lead to a paradox, so-called the "compactness problem". If one assumes that a photonic energy of $10^{52} \mathrm{erg}$, distributed according to the GRB spectrum, is released in a small volume of linear dimensions $R \leq c \delta t$, then the optical depth to pair creation is $\tau \sim 10^{15}$. If that was true, all the photons would have interacted to create pairs and thermalize. However, the observed spectrum of GRBs is highly nonthermal!

The only known solution to the "compactness problem" is relativistic motion. If the emission site is moving toward the observer with a relativistic speed characterized by a Lorentz factor $\gamma$, then the optical depth is reduced compared to the stationary estimate, due to two effects. First, the size of the source can be larger by a factor of $\gamma^{2}$. This will still produce variability over a short time scale given by $\delta T=R / \gamma^{2} c$, since the radiation from a relativistically moving source is beamed. Second, the photons in the local frame are softer by a factor of $\gamma$, and, therefore, only a small fraction of them at the high-energy tail have enough energy to create pairs. The combination of these two effects reduces the optical depth by a factor of $\sim \gamma^{6.5}$. Therefore, the optical depth is reduced below unity, and the "compactness problem" is solved, if the Lorentz factor is larger than about a hundred.

This solution led to a three-stage generic scenario for GRBs. First, a compact source releases about $10^{52} \mathrm{erg}$ in a small volume of space and on a short time scale. Then, this large concentration of energy expands due to its own pressure. If the rest mass that contaminates the site is not too large $\left(\leq 10^{-5} M_{\odot}\right)$, this will result in relativistic expansion with $\gamma>100$. Finally, at a large enough radius, the kinetic energy of the expanding material is converted to internal energy and radiated mainly in $\gamma$-rays. At this stage, the system is optically thin and high-energy photons can escape. 
This three-stage process has one disappointing property: we do not see directly the inner engine. This is one of the reasons that this source is still so mysterious. Despite this fact, we will show that the temporal structure of the observed bursts resembles that of the source. In this talk, we will concentrate mainly on the third stage. We will assume that a relativistic flow with a high Lorentz factor exists, carrying more than $10^{52} \mathrm{erg}$ as kinetic energy, and discuss how this flow may produce the $\gamma$-ray photons as well as the afterglow. This presentation will be short in equations, stressing the main, qualitative ideas.

\section{Internal Versus External Shocks}

Assume a flow carrying $10^{52} \mathrm{erg}$ as kinetic energy. In order for this to produce photons, the kinetic energy must be converted back into internal energy and radiated away. The flow must therefore, at least partially, slow down. Two scenarios were proposed for this deceleration: external shocks (Mészáros \& Rees 1993) and internal shocks (Narayan, Paczyński, \& Piran 1992; Rees \& Mészáros 1994). In the external shocks scenario, the relativistic material is running into some (external) ambient medium, probably the interstellar medium or a wind that was emitted earlier by the progenitor. In the internal shocks scenario, the inner engine is assumed to emit an irregular flow that consists of many shells that travel with a variety of Lorentz factors and therefore collide into each other and thermalize some of their kinetic energy.

The property that proved to be very useful in constraining these two possibilities is the variability observed in many of the bursts. In the external shocks scenario, this variability is attributed to irregularities in the surrounding medium, e.g., clouds. Each time the ejecta run into a higher density environment, it produces a peak. In the internal shocks scenario, the source has to emit many shells, and whenever two of them collide, a peak is produced. External shocks require a complicated surrounding with a relatively simple source that explodes once, while internal shocks require a more complicated source that will explode many times to produce several shells. Due to these very different requirements on the source, the question of internal or external shocks is of fundamental importance in understanding the basic nature of the phenomenon.

As discussed by Fenimore, Madras, \& Nayakshine (1996), the size of the clouds in which the ejecta runs into, in the external shocks scenario, has to be very small to produce peaks that are narrower than the duration of the burst. Sari \& Piran (1997) gave the following argument. The size of the clouds has to be smaller than $R / N \gamma$ to produce peaks that are narrower by a factor of $N$ than the duration of the burst. The number of such clouds should be smaller than $N$, otherwise, pulses arriving from different clouds will overlap and the amplitude of the variability will be reduced. Finally, the observable area of the ejecta, due to relativistic beaming, is $(R / \gamma)^{2}$. The maximal efficiency of the external shocks scenario is therefore given by

$$
\frac{\text { cloud area } \times \text { number of clouds }}{\text { observed shell area }} \leq \frac{1}{N} \sim 1 \% \text {. }
$$

Since, in many bursts, $N>100$, external shocks have a severe efficiency problem when constructed to produce highly variable bursts. More detailed studies 

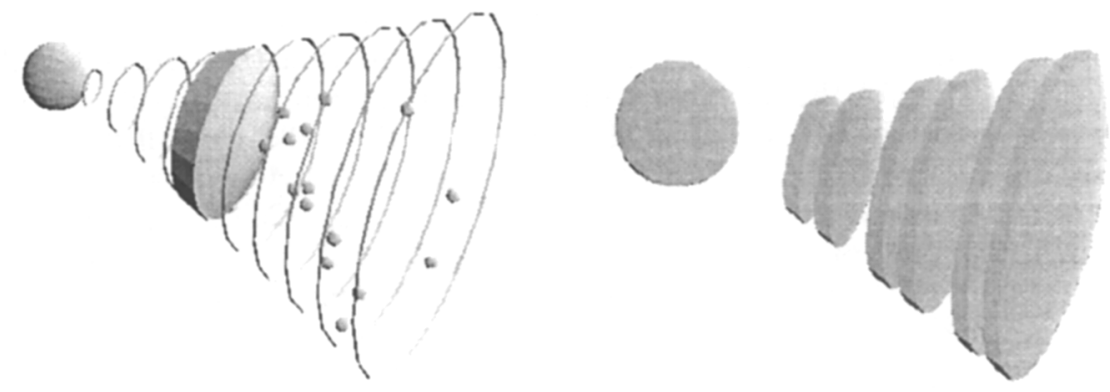

Figure 2. Producing variability by external shocks (left) or internal shocks (right). The external shocks scenario has low efficiency.

(Ramirez-Ruiz \& Fenimore 1999) have shown that other predictions of the external shock are also inconsistent with the observed temporal profile. Moreover, the density ratio between the cloud and the surrounding has to be huge, of the order of $\gamma N^{2} \sim 10^{6}$, in order that the ejecta will be slowed down mainly by the dense clouds rather than by the low-density, uniform medium.

Internal shocks, on the other hand, do not suffer from these problems. The variability can be produced even without breaking the spherical symmetry. Detailed calculations show that the observed temporal structure coming from internal shocks closely follows the operation of the inner engine that generated the shells (Kobayashi, Piran, \& Sari 1997). In this scenario, the source must be variable on time scales shorter than a second and last for as long as 100 seconds, just as the bursts themselves do.

\section{The Afterglow Revolution}

The study of $\gamma$-ray bursts was revolutionized when the Italian-Dutch satellite BeppoSAX delivered arcminute positioning of some GRBs, within a few hours time scale. This enabled other ground and space instruments to monitor the relatively narrow error box. Emission in X-ray, infrared, optical, and radio, socalled "afterglow", was observed by now for about a dozen bursts. The study of GRBs, that was up until then collimated to a narrow energy band, immediately turned into a multiwavelength astronomy field. Due to the transient nature of the afterglow, a major part of the game is to observe the GRBs field early enough when the afterglow is still bright. Within the first day, the optical emission is usually brighter than $20^{\text {th }}$ magnitude, and therefore, small telescopes can play an important role in measuring the light curve. A large, worldwide collaboration is observing these events, and the data are submitted to an impressive Global-Coordinate-Network (Barthelmy et al. 1994) in real time, allowing other observatories to react accordingly. At late times, the decaying counterpart becomes fainter than its host galaxy, and HST is used to measure its optical flux.

The observed afterglow usually shows a power law decay $t^{-\alpha}$ in the optical and $\mathrm{X}$-rays, where a typical value is $\alpha \cong 1.2$. Some afterglows show a steeper decline with $\alpha \cong 2$. At radio wavelengths, the flux seems to rise on time scales 

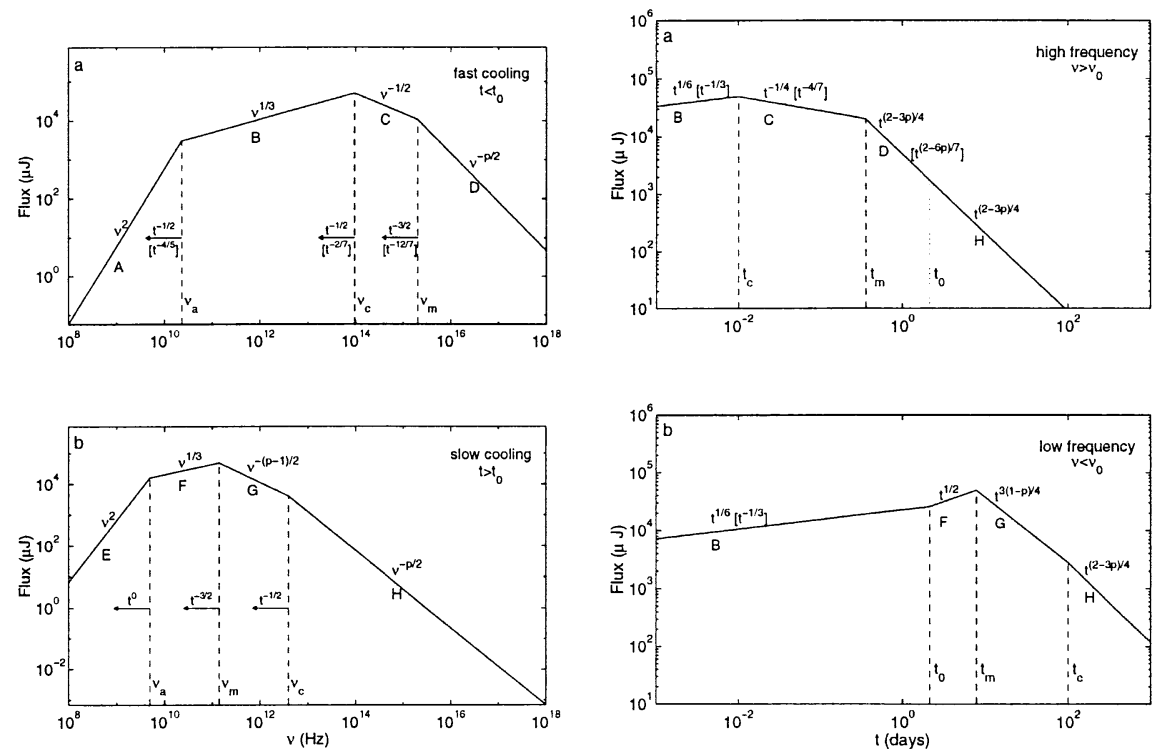

Figure 3. Theoretical spectra (left) and light curves (right) of synchrotron emission from a power law distribution of electrons. See SPN for details. The observed spectra and light curves are fit well by $p=$ $2.2-2.4$.

of weeks and then decay with a similar power law. In some cases, the radio flux was observed for about a year following the few-seconds GRB.

\section{Afterglow - Basic Theory}

The afterglow was predicted well before it was observed (Paczyński \& Rhoads 1993; Katz 1994; Vietri 1997; Mészáros \& Rees 1997). After the internal shocks produce the GRB, the shell interacts with the surrounding medium and decelerates. The emission shifts into lower and lower frequencies. Excitingly, the afterglow theory is relatively simple. It deals with the emission on time scales much longer than those of the GRBs. The details of the complex initial conditions are therefore forgotten, and the description depends on a small number of parameters, such as the total energy and the external density.

The basic model assumes that electrons are accelerated by the shock into a power law distribution, $N\left(\gamma_{e}\right) \sim \gamma_{e}^{-p}$ for $\gamma_{e}>\gamma_{m}$. The lower cutoff of this distribution is assumed to be a fixed fraction of equipartition. It is also assumed that a considerable magnetic field is being built behind the shock; it is again characterized by a certain fraction $\epsilon_{B}$ of equipartition. The relativistic electrons then emit synchrotron radiation, which is the observed afterglow. The broadband spectrum of such emission was given by Sari, Piran, \& Narayan (1998).

At each instant, there are three characteristic frequencies. (I) The synchrotron frequency of the minimal energy electron is $\nu_{m}$, having a Lorentz factor $\gamma_{m}$. (II) The cooling time of an electron is inversely proportional to its 
Lorentz factor $\gamma_{e}$. Therefore, electrons with a Lorentz factor higher than a critical Lorentz factor $\gamma_{e}>\gamma_{c}$ can cool on the dynamical time scale of the system. This characteristic Lorentz factor corresponds to the "cooling frequency" $\nu_{c}$. (III) Below some critical frequency $\nu_{a}$, the flux is self-absorbed and is given by the Rayleigh-Jeans portion of a blackbody spectrum. The broadband spectrum of the well-studied GRB 970508 (Galama et al. 1998) is in very good agreement with the theoretical picture.

The evolution of this spectrum as a function of time depends on the hydrodynamic evolution. The simplest, which also well describes the data, is the adiabatic model with a constant-density, surrounding medium. The rest mass collected by the shock at radius $R$ is about $R^{3} \rho$. On the average, the particles move with a Lorentz factor of $\gamma^{2}$ in the observer frame, and therefore, the total energy is given by $E \sim \gamma^{2} R^{3} \rho c^{2}$. Assuming that the radiated energy is negligible compared to the flow energy, we obtain that $\gamma \sim R^{-3 / 2}$, or, in terms of the observer time, $t=R / \gamma^{2} c$, we get $\gamma \sim t^{-3 / 8}$. If, on the other hand, the density drops as $R^{-2}$ (as is expected if the surrounding is a wind produced earlier by the progenitor of the burst), we get $\gamma \sim t^{-1 / 4}$. These simple scaling laws lead to the spectrum evolution as given in Figure 3. Given the above hydrodynamic evolution, one can construct light curves at any given frequency. These will also consist of power laws, changing from one power law to the other once the break frequencies sweep through the observed band. These power laws are in fair agreement with the afterglow observations.

\section{Jets and Beaming}

The hydrodynamic evolution described above assumed spherical symmetry. Scenarios in which the ejecta are limited to a solid angle $\Omega=\pi \theta_{0}^{2}$ are usually called "jets". These "jets" should not be confused with the relativistic beaming of the radiation. The term "jet" corresponds to the physical shape of the outflow and is created by the inner engine. In contrast, the relativistic beaming is a special relativity effect and has to do only with the fact that the ejecta are moving with relativistic Lorentz factor $\gamma$. The relativistic beaming allows an observer to see only a small angular extent of size $1 / \gamma$ centered around the line of sight.

The question of "jets" has two important implications. First, the true total energy emitted by the source is smaller by a factor of $\Omega / 4 \pi \sim \theta_{0}^{2} / 4$ than if the ejecta surface was spherical. Second, the event rate must be bigger by the same factor to account for the observed rate.

Interestingly, due to the relativistic beaming (which is independent of jets), we are only able to see an angular extent of $1 / \gamma<0.01$ during the GRB itself. Therefore, we cannot distinguish a jet from a spherical ejecta surface. Therefore, given the bursts only, the event rate and the energy in each GRB are unknown to about four orders of magnitude! However, as $\gamma$ decreases, it will eventually fall below the inverse opening angle of the jet. The observer will appreciate that some of the sphere is missing by the fact that less radiation is observed. This effect will produce a significant break, steepening the light curve decay by a factor of $\gamma^{2} \sim t^{-3 / 4}$. The transition should occur when $1 / \gamma=\theta_{0}$, and it therefore provides an indication for the jet's opening angle. Additionally, Rhoads (1999) has shown that, at about the same time, the physical size of the jet will begin 

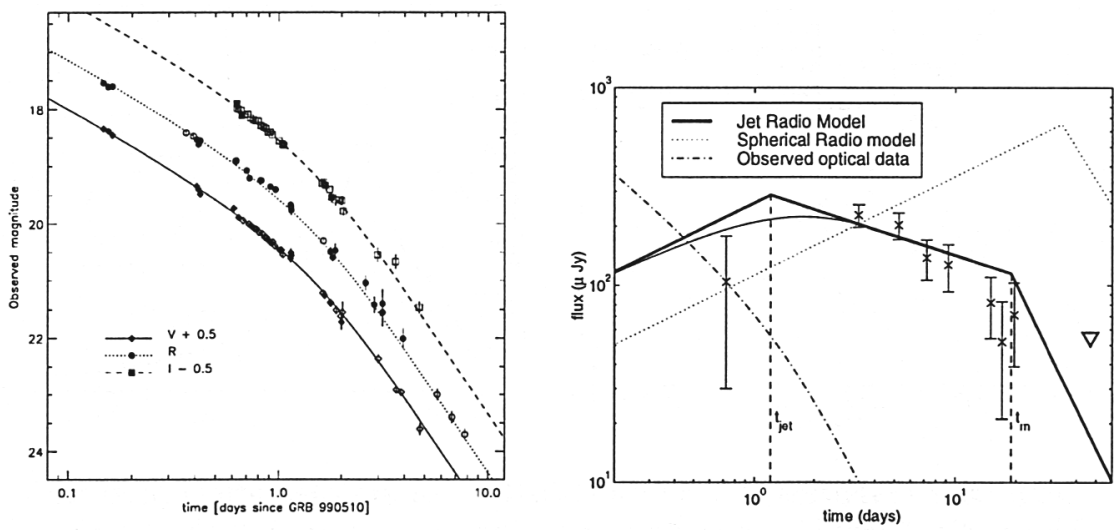

Figure 4. GRB 990510, the best evidence for a "jet": an achromatic break in optical and radio at $t_{j e t} \cong 1.2$ days, implying $\theta_{0} \cong 0.08$.

to increase so that $\theta(t) \sim 1 / \gamma$. Taking this effect into account, the break is even more significant, and the decay is proportional to $t^{-p} \sim t^{-2.2}-t^{-2.4}$.

Evidence of a break from a shallow to a steep power law was seen in GRB 990123 (Kulkarni et al. 1999a; Fruchter et al. 1999). Unfortunately, the break was observed only in one optical band, while the data in other bands were ambiguous. A very clear break was seen in GRB 990510 (Stanek et al. 1999; Harrison et al. 1999) simultaneously in all optical bands and radio. In GRB 990123 and GRB 990510, the transition times were $\sim 2.1$ days and 1.2 days, reducing the isotropic energy estimate by a factor of $\sim 200$ and $\sim 300$, respectively.

Sari, Piran, \& Halpern (1999) have noted that the observed decays in GRB afterglows that do not show a break are either of a shallow slope of approximately $t^{-1.2}$ or a very steep slope of approximately $t^{-2}$. They argued that the rapidly decaying bursts are those in which the ejecta were in a narrow jet and the break in the light curve occurred before the observations. Interestingly, evidence for jets is found when the independently inferred energy is largest. This implies that the jets account for a considerable fraction of the wide luminosity distribution seen in GRBs, and that the true energy distribution is less wide than it seems.

\section{GRB 990123: The Optical Flash and the Radio Flare}

An exiting event this year was the first detection of a bright ( (th $^{\text {th }}$ magnitude) optical emission simultaneous with GRB 990123 (Akerlof 1999). A theoretical prediction for such a flash was recently given in detail by Sari \& Piran (1999a, b) and was earlier suggested as a possibility by Mészáros \& Rees (1997). During the first few tens of seconds, the evolution of the Lorentz factor as a function of time is not self-similar. There are two shocks: a forward shock going into the surrounding medium and a reverse shock going into the expanding shell. The hydrodynamic details were discussed by Sari \& Piran (1995). During the initial stage, the internal energies stored behind the shocked surrounding matter and the shocked ejecta are comparable. However, the temperature of the shocked 

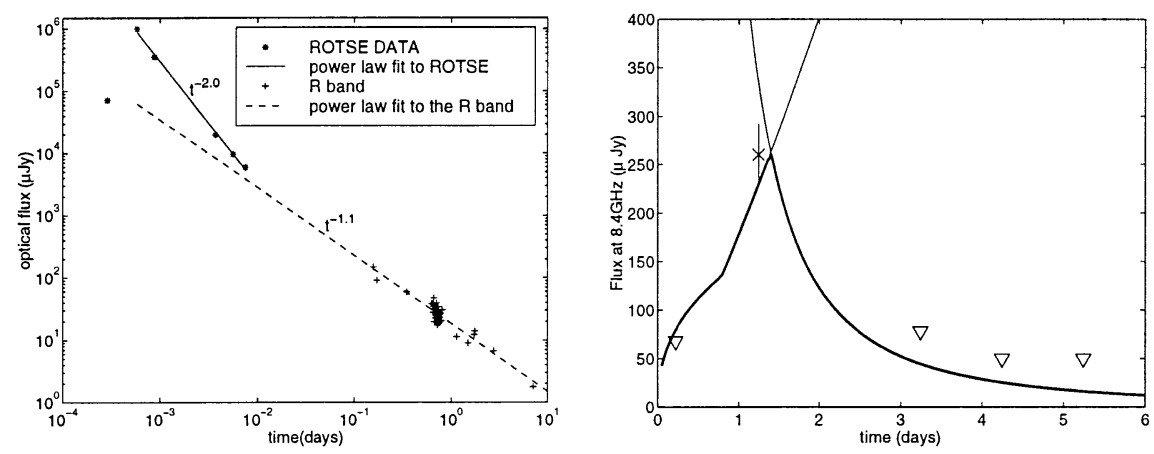

Figure 5. GRB990123: Optical (left) data fits theoretical prediction. The radio "flare" seen a day after the burst agrees with theoretical scaling of the optical data (heavy solid line).

ejecta is much lower, typically by a factor of $\gamma \sim 10^{2}$. This results in additional emission with a typical frequency lower by a factor of $\gamma^{2} \sim 10^{4}$, which, for typical parameters, falls in the optical regime. Contrary to the "standard" late afterglow, this emission is very sensitive to the initial Lorentz factor.

The optical flash of GRB 990123 peaked $\sim 60$ s after the burst's trigger. Sari \& Piran (1999c) and Mészáros \& Rees (1999) have shown that the observed optical emission can be well described by the emission from the reverse shock that initially decelerates the ejecta, provided the initial Lorentz factor is about 200. It takes tens of seconds for the reverse shock to sweep through the ejecta and produce the bright flash. Later, the shocked, hot matter expands adiabatically, and the emission quickly shifts to lower frequencies and considerably weakens.

Another new ingredient that was found in GRB 990123 is a radio flare (Kulkarni et al. 1999b). Contrary to all other afterglows, where the radio peaks after a few weeks and then decays slowly, this burst had a fast rising flare, peaking at around a day and decaying quickly. Sari \& Piran (1999c) have shown that within a day the emission from the adiabatically cooling ejecta that produced the $60 \mathrm{~s}$ optical flash shifts into the radio frequencies. The optical flash and the radio flare are therefore related. The fact that the "usual" forward-shock radio emission did not show up later, after a time scale of weeks, is in agreement with the interpretation of this burst as a "jet" which causes the emission to considerably weaken by the time the frequency arrives to the radio.

\section{Polarization - A Promising Tool}

An exciting possibility to further constrain the models and obtain a direct proof of the geometrical picture of "jets" is to measure polarization. Gruzinov \& Waxman (1999) and Medvedev \& Loeb (1999) considered the emission from spherically ejected material, which by symmetry should produce no polarization on the average. Polarization is more natural if the ejecta is a "jet" and the observer is not directed at its very center (Gruzinov 1999; Ghisellini \& Lazzati 1999; Sari 1999), since the spherical symmetry is broken. For simplicity, assume 

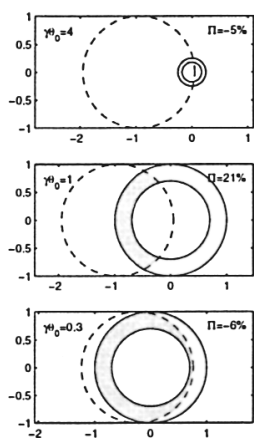
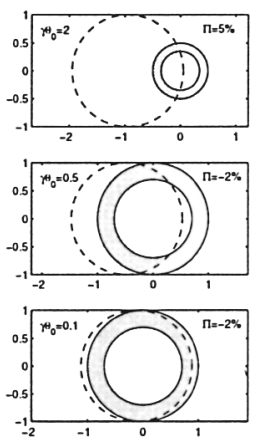

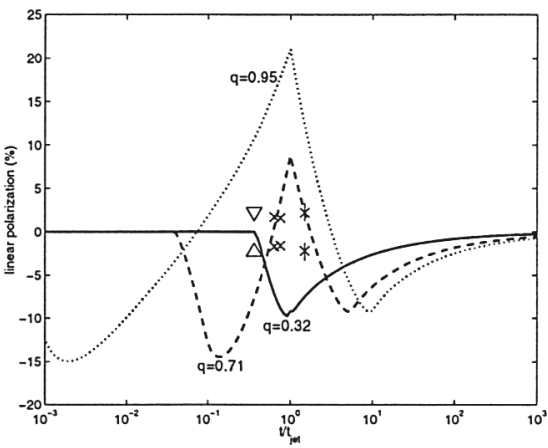

Figure 6. Left: Evolution of the observed ring (gray) and the physical jet (dashed). Right: observed and theoretical polarization light curve.

that the components of the magnetic field in the shock plane are larger than its perpendicular component (the results are more general, unless the magnetic field has no preferred direction). The synchrotron polarization from each part of the fireball, which is perpendicular to the magnetic field, is directed radially.

As long as the relativistic beaming of size $1 / \gamma$ is narrower than the physical size of the jet $\theta_{0}$, one is able to see a full ring, and therefore, the radial polarization averages to zero. As the flow decelerates, the relativistic beaming $1 / \gamma$ becomes comparable to $\theta_{0}$, and only a part of the ring is visible. Net polarization is then observed. Note that due to the radial direction of the polarization from each fluid element, the total polarization is maximal when a quarter or three quarters of the ring are missing (or radiate less efficiently), and vanishes for a full or half ring. The observed polarization when more than half of the ring is missing is perpendicular to the direction when less than half of it is missing.

At late stages, the jet expands, and since the offset of the observer from the physical center of the jet is constant, spherical symmetry is regained. The vanishing and re-occurrence of significant parts of the ring results in a unique prediction: there should be three peaks of polarization, with the polarization position angle during the middle peak rotated by $90^{\circ}$ with respect to the other two peaks. In the case that the observer is very close to the center, more than half of the ring is always observed, and therefore, only a single direction of polarization is expected. A few possible polarization light curves are presented in the Figure 6.

\section{Summary - What Does it all Teach us About the Source Itself?}

(i) Internal shocks imply that the source is variable on $<1 \mathrm{~s}$ time scales but lasts for tens of seconds. (ii) The event rate is probably higher than observed by about a factor of a hundred since some events are narrow jets. This translates to one event per $10^{5}$ years per galaxy. (iii) The environment of at least some bursts agrees well with ordinary ISM densities. These bursts do not occur in their galaxies' halos. (iv) Measurements of optical flashes and radio flares probe the ejecta material, allowing us to measure the number of baryons which reside 
in the explosion site. GRB 990123 has $\gamma_{0} \sim 200$. (v) Taking jets into account, the total energy involved can be "only" $10^{52} \mathrm{erg}$, even in the most extreme case.

\section{References}

Akerlof, C., et al. 1999, Nature, 398, 400

Band, D., et al. 1993, ApJ, 413, 281

Barthelmy, S. D., et al. 1994, in AIP Conf. Proc. 307, Second Huntsville Workshop, eds. G. Fishman, J. Brainerd, \& K. Hurley (New York: AIP), 643

Fenimore, E. E., Madras, C., \& Nayakshine, S. 1996, ApJ, 473, 998

Fruchter, A. S., et al. 1999, ApJ, 519, L13

Galama, T. J., et al. 1998, ApJ, 500, 101

Ghisellini, G., \& Lazzati, D. 1999, MNRAS, 309, L7

Gruzinov, A. 1999, ApJ, 525, L29

Gruzinov, A., \& Waxman, E. 1999, ApJ, 511, 852

Harrison, F. A., et al. 1999, ApJ, 523, L121

Katz, J. I. 1994, ApJ, 422, 248

Klebesadel, R. W., Strong, I. B., \& Olson, R. A. 1973, ApJ, 182, 85

Kobayashi, S., Piran, T., \& Sari, R. 1997, ApJ, 490, 92

Kulkarni, S. R., et al. 1999a, Nature, 398, 389

Kulkarni, S. R., et al. 1999b, ApJ, 522, L97

Medvedev, M. V., \& Loeb, A. 1999, astro-ph/9904363, preprint

Mészáros, P., \& Rees, M. J. 1993, ApJ, 405, 278

Mészáros, P., \& Rees, M. J. 1997, ApJ, 476, 232

Mészáros, P., \& Rees, M. J. 1999, MNRAS, 306, L39

Narayan, R., Paczyński, B., \& Piran, T. 1992, ApJ, 395, 83

Paciesas, W. S., et al. 1999, ApJS, 122, 465

Paczyński, B., \& Rhoads, J. 1993, ApJ, 418, L5

Ramirez-Ruiz, E., \& Fenimore, E. E. 1999, A\&A, 138, 521

Rees M. J., \& Mészáros, P. 1994, ApJ, 430, L93

Rhoads, J. E. 1999, ApJ, 525, 737

Sari, R. 1999, ApJ, 524, L43

Sari, R., \& Piran, T. 1995, ApJ, 455, L143

Sari, R., \& Piran, T. 1997, ApJ, 485, 270

Sari, R., \& Piran, T. 1999a, A\&A, 138, 537

Sari, R., \& Piran, T. 1999b, ApJ, 520, 641

Sari, R., \& Piran, T. 1999c, ApJ, 517, L109

Sari, R., Piran, T., \& Halpern, J. 1999, ApJ, 519, L17

Sari, R., Piran, T., \& Narayan, R. 1998, ApJ, 497, L17

Stanek, K. Z., Garnavich, P. M., Kaluzny, J., Pych, W., \& Thompson, I. 1999, ApJ, 522, L39

Vietri, M. 1997, ApJ, 478, L9 